\title{
Modulation Multiplexing Distributed Space-Time Block Coding for Two-User Cooperative Diversity in Wireless Network
}

\author{
Rong Ran and Dongku Kim \\ Department of Electrical and Electronic Engineering, Yonsei University, Seoul, \\ 120-749 Korea \\ \{sunny_rr,dkkim\}@yonsei.ac.kr
}

\begin{abstract}
In this paper, we propose a modulation multiplexing distributed space-time coding for two-user cooperative diversity in wireless network, in which the information of one user lies in the in-phase axis (I axis), while that of the other user lies in quadrature-phase axis ( $\mathbf{Q}$ axis). Since two users share both time and frequency resource in cooperation sub-frame, the proposed scheme is more bandwidth efficient. We characterize performance of the proposed scheme in symmetric interuser case. Simulation results show that the proposed scheme outperforms the amplify-to-forward cooperative system. Compared to the distributed space-time coding with time-division multiple accesses cooperative system, the proposed scheme achieves the same diversity gain but loses some coding gain. However, when the selection relay protocol is adopted for the proposed scheme, coding gain is also achieved.
\end{abstract}

Keywords: Distributed Space-Time Coded Cooperative diversity system, Alamouti structure, Modulation Multiplexing.

\section{Introduction}

In wireless networks, signal fading arising from multipath propagation is a particularly severe form of interference that can be mitigated through the use of diversity-transmission of redundant signals over essentially independent channel realizations in conjunction with suitable receiver combining to average the channel effects. Traditional space-time coded multi-antenna system exploits spatial diversity through multiple transmit or receive antennas. However, due to the size of mobile and carrier frequency constraints, achieving spatial diversity through multiple antennas may not be possible. In this case, a promising approach for achieving spatial diversity without multiple antennas is cooperative diversity. As there are many users in the wireless system, cooperative diversity allows sharing the resource (e.g., time, frequency) among cooperative users and each user can serve as a relay for the transmission of other user's information. Diversity is obtained from the relay transmission of other users in the system. The concept of cooperative diversity was introduced in [1, where the capacity region, outage probabilities, coverage area were derived and code-division multiple-access 
(CDMA) implementation was discussed. In [2] and [3], various cooperative diversity algorithms are developed for a pair of users based upon relays amplifying their received signals or fully decoding and repeating information. These algorithms are referred as amplify-and-forward and decode-and-forward, respectively. Distributed space-coded cooperative (DSTC) systems were introduced to combat fading in [4] and [5]. In these systems, a pair of users cooperates in order to construct the space-time coded transmission. Full spatial diversity benefits of these DSTC systems come at a price of decreasing bandwidth efficiency with the number of cooperation users, because each relay requires its own time or frequency resource to relay the partner's information. The analysis of these systems was given in [6], in which the outage probability is a performance measure. The idea of using distributed space-time coding has also been reported in [7].

In this paper, a situation that both users have information to send and they share the same frequency band is considered. Therefore, we propose a modulation multiplexing Distributed space time coding (DSTC-MM) for two-user cooperative diversity system. In this system, The information of one user lies in the in-phase axis (I axis), while that of the other user lies in the quadrature-phase axis ( $\mathrm{Q}$ axis), which is to ensure that both users can share time and frequency resource in cooperation subframe so as to increase the bandwidth efficiency. We focus on a half-duplex transmission, i.e. the users cannot transmit and receive signal at the same time.

\section{System and Signal Model}

We consider a cellular system in which two cooperative users transmit their information to the same base station (BS) shown as in Fig.1. A single antenna is accommodated at each user and BS. The transmission frame structure and its associated transmissions and reception are shown in Fig[1. A total transmission frame is divided into three sub-frames. The first sub-frame belongs to the first user (U1). The second sub-frame belongs to the second user (U2). The cooperation sub-frame is shared by both users and used to relay each other's message to the destination. Compared with the distributed space-time coding with TDMA cooperative system [6], this partition is more bandwidth efficient since cooperation transmissions share both time and frequency resource. In order

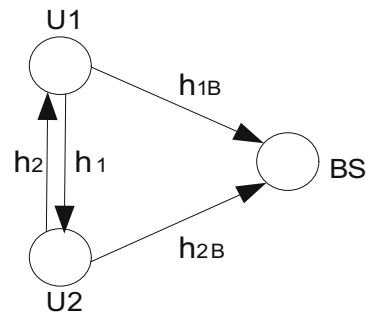

Fig. 1. System Model 


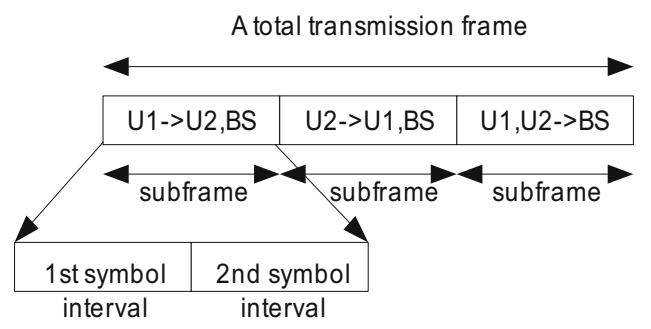

Fig. 2. Transmission frame structure

to apply space-time block code transmission, we assume each sub-frame includes two symbol intervals.

We assume that the inter-user channels and the user-destination channels are independent of each other. All channels experience quasi static flat fading, i.e., they are fixed during a sub-frame and change independently in the next subframe. With the above assumption, we consider a discrete-time model. In the first sub-frame, the first user broadcasts the signal vectors $\mathbf{s}_{1}[i]=\left(s_{1}[2 i], s_{1}[2 i+1]\right)$ of equally probable MPAM sysbols lied in $\mathbf{I}$ axis. At BS, the received signal vector $\mathbf{r}_{1 B}$ corresponding to the direct transmission path from the first user to $\mathrm{BS} \mathbf{r}_{1 B}[i]=\left(r_{1}[2 i], r_{1}[2 i+1]\right)$ is given as

$$
\mathbf{r}_{1 B}=\left(r_{1}[2 i] r_{1}[2 i+1]\right)=h_{1 B}[i] \cdot\left(s_{1}[2 i] s_{1}[2 i+1]\right)+\left(n_{1 B}[2 i] n_{1 B}[2 i+1]\right)
$$

where the channel gain, $h_{m B}[i], m \in\{1,2\}$, are associated with a transmission path from the $m$ th user to BS at the $i$ th sub-frame. $n_{m B}[k], m \in\{1,2\}$, representing additive white noise and interference in the path from the $m$ th user to the base station at the $k$ th symbol interval, is modeled as independent circularly symmetric zero-mean complex Gaussian random variables with variances $N$. Meanwhile, the received signal vector for the second user $\mathbf{r}_{12}[i]=\left(r_{12}[2 i], r_{12}[2 i+1]\right)$ is given as

$$
\mathbf{r}_{12}=\left(r_{12}[2 i] r_{12}[2 i+1]\right)=h_{1}[i] \cdot\left(s_{1}[2 i] s_{1}[2 i+1]\right)+\left(n_{12}[2 i] n_{12}[2 i+1]\right)
$$

where $h_{m}[i], m \in\{1,2\}$, represents channel gain of the path from the $m$ th user to its partner at $i$ th sub-frame. And $n_{12}[k]$ represents additive white noise and interference in the path from the first user to its partner at $k$ th symbol interval.

In the second sub-frame, the second user's information lies on the $\mathbf{Q}$ axis, i.e. $\mathbf{s}_{2}[i]=\left(j \cdot s_{2}[2 i], j \cdot s_{2}[2 i+1]\right)$ and $j=\sqrt{-1}$. The roles of the first and second users are switched. Therefore, the received signal vector at BS and the first user are $\mathbf{r}_{2 B}[i]=\left(r_{2}[2 i], r_{2}[2 i+1]\right)$ and $\mathbf{r}_{21}[i]=\left(r_{21}[2 i], r_{21}[2 i+1]\right)$ respectively. where

$\mathbf{r}_{2 B}=\left(r_{2}[2 i] r_{2}[2 i+1]\right)=h_{2 B}[i] \cdot\left(j \cdot s_{2}[2 i] j \cdot s_{2}[2 i+1]\right)+\left(n_{2 B}[2 i] n_{2 B}[2 i+1]\right)$ 
and

$\mathbf{r}_{21}=\left(r_{21}[2 i] r_{21}[2 i+1]\right)=h_{2}[i] \cdot\left(j \cdot s_{2}[2 i] j \cdot s_{2}[2 i+1]\right)+\left(n_{21}[2 i] n_{21}[2 i+1]\right)$

The signal-to-noise ratios (SNRs) associated with the user-destination link are defined as $\operatorname{snr}_{n B}[k]=E\left|h_{n B}[k]\right|^{2} / N, n \in\{1,2\}$. For simplicity in the comparison, both SNRs for the inter-user channel are set to be equal and defined as $i s n r[k]=E\left|h_{1}[k]\right|^{2} / N=E\left|h_{2}[k]\right|^{2} / N$.

\section{Modulation Multiplexing DSTC(DSTC-MM)}

During the cooperation sub-frame. The same amplify-and-forward protocol as that in 2] is applied in this paper. For the first user, the transmitted signal vector constructed by the relay information and its own information is given as follows

$$
\begin{aligned}
\mathbf{s}_{1}[i+1]= & \left(s_{1}[2 i+2] s_{1}[2 i+3]\right) \\
& =\sqrt{\alpha_{1}} \cdot\left(\left(\left|h_{2}[i]\right| s_{1}[2 i]+\sigma_{2} r_{21}[2 i+1]\right)\left(\left|h_{2}[i]\right| s_{1}[2 i+1]+\sigma_{2} r_{21}[2 i]\right)\right)
\end{aligned}
$$

where $\sigma_{i}, i \in\{1,2\}$ is an automatic gain control (AGC) for a non-regenerative system, which is required at the relay mobile in order to prevent $r_{m n}[k]$ from saturating the relay amplifier. In this paper, we adopt an AGC that employs

$$
\sigma_{k}=\frac{h_{k}^{*}[i]}{\left|h_{k}[i]\right|}, k \in\{1,2\}
$$

for the second user, the transmitted signal vector is shown as follows

$$
\begin{aligned}
\mathbf{s}_{2}[i+1]= & \left(s_{2}[2 i+2] s_{2}[2 i+3]\right) \\
& =\sqrt{\alpha_{2}} \cdot\left(\left|h_{1}[i]\right| j s_{2}[2 i]+\sigma_{1} r_{12}[2 i+1]\left|h_{1}[i]\right| j s_{2}[2 i+1]+\sigma_{1} r_{12}[2 i]\right)
\end{aligned}
$$

These signals are transmitted by using the Alamouti-like structure. At the base station, the received signal vector is

$$
\begin{aligned}
\mathbf{r}[i+1]= & \left(r_{B}[2 i+2] r_{B}[2 i+3]\right) \\
& =\left(\begin{array}{c}
h_{1 B}[i+1] \\
h_{2 B}[i+1]
\end{array}\right)^{T}\left(\begin{array}{cc}
s_{1}[2 i+2] & s_{1}[2 i+3] \\
-s_{2}^{*}[2 i+2] & s_{2}^{*}[2 i+3]
\end{array}\right)+\left(\begin{array}{c}
n_{B}[2 i+2] \\
n_{B}[2 i+3]
\end{array}\right)^{T}
\end{aligned}
$$

By complex conjugation of the second element of $\mathbf{r}[i+1]$, the input/output relation can be reformulated as follows:

$$
\begin{aligned}
\mathbf{r}[i+1]= & \left(\begin{array}{c}
r_{B}[2 i+2] \\
r_{B}^{*}[2 i+3]
\end{array}\right) \\
& =\mathbf{H}\left(\begin{array}{c}
s_{1}[2 i]+j \cdot s_{2}[2 i+1] \\
s_{1}[2 i+1]+j \cdot s_{2}[2 i]
\end{array}\right)+\text { Noise }
\end{aligned}
$$


where

$$
\mathbf{H}=\left(\begin{array}{c}
\sqrt{\alpha_{1}} h_{1 B}[i+1]\left|h_{2}[i]\right|-\sqrt{\alpha_{2}} h_{2 B}[i+1]\left|h_{1}[i]\right| \\
\sqrt{\alpha_{2}} h_{2 B}^{*}[i+1]\left|h_{1}[i]\right| \\
\sqrt{\alpha_{1}} h_{1 B}^{*}[i+1]\left|h_{2}[i]\right|
\end{array}\right)
$$

and

$$
\text { Noise }=\left(\begin{array}{c}
\frac{\sqrt{\alpha_{1}} h_{1 B}[i+1] h_{2}^{*}[i] n_{21}[2 i+1]}{\left|h_{2}[i]\right|}-\frac{\sqrt{\alpha_{2}} h_{2 B}[i+1] h_{1}^{*}[i] n_{12}^{*}[2 i+1]}{\left|h_{1}[i]\right|} \\
\frac{\sqrt{\alpha_{1}} h_{1 B}[i+1] h_{2}^{*}[i] n_{21}[2 i+1]}{\left|h_{2}[i]\right|}+\frac{\sqrt{\alpha_{2}} h_{2 B}[i+1] h_{1}^{*}[i] n_{12}^{*}[2 i+1]}{\left|h_{1}[i]\right|}
\end{array}\right)+\left(\begin{array}{l}
n_{B}[2 i+2] \\
n_{B}^{*}[2 i+3]
\end{array}\right)
$$

The received signals in (9) are similar to those of the Alamout's scheme. The difference is an additive noise in (11). Therefore, the receiver detects

$$
\begin{aligned}
\hat{\mathbf{r}}[i+1]= & \left(\begin{array}{c}
\hat{r}_{B}[2 i+2] \\
\hat{r}_{B}[2 i+3]
\end{array}\right)=\mathbf{H}^{H} \mathbf{r}[i+1] \\
& =\left(\left|h_{1 B}[i+1] h_{2}[i]\right|^{2} a_{1}+\left|h_{2 B}[i+1] h_{1}[i]\right|^{2} a_{2}\right)\left(\begin{array}{c}
s_{1}[2 i]+j s_{2}[2 i+1] \\
\left(s_{1}[2 i+1]+j s_{2}[2 i]\right)^{*}
\end{array}\right) \\
& +\mathbf{H}^{H} \cdot \text { Noise }
\end{aligned}
$$

Since the symbols $s_{1}\left([k]\right.$ and $j s_{2}[k]$ are in $\mathbf{I}$ axis and $\mathbf{Q}$ axis respectively, we can decode them separately by considering the real and imaginary parts of (12), therefore, the following descriptions are obtained:

$$
\begin{aligned}
\hat{s}_{1}[2 i] & =\operatorname{Re}\left(\hat{r}_{B}[2 i+2]\right) \\
\hat{s}_{1}[2 i+1] & =\operatorname{Re}\left(\hat{r}_{B}[2 i+3]\right) \\
\hat{s}_{1}[2 i] & =\operatorname{Im}\left(\hat{r}_{B}[2 i+3]\right) \\
\hat{s}_{1}[2 i+1] & =\operatorname{Im}\left(\hat{r}_{B}[2 i+2]\right)
\end{aligned}
$$

Furthermore, $r_{k B}[2 i]$ and $\hat{s}_{k}[2 i], k \in\{1,2\}$ do not share the same noise power, we use a Maximum Ratio Combiner (MRC) to optimally combine these two signals, and each branch of the MRC receiver is weighted by its respective complex fading gain over the total noise power on that particular branch. The output of the MRC is shown as follows

$$
\tilde{s}_{k}[2 i]=w_{k}[i+1] \hat{s}_{k}[2 i]+w_{k}[i] r_{k B}[2 i]
$$

where

$$
w_{k}[i+1]=\frac{2}{N} \frac{1}{\left|h_{1 B}[i+1]\right|^{2}+\left|h_{2 B}[i+1]\right|^{2}+1}, w_{k}[i]=\frac{h_{k B}^{*}[i]}{N}
$$

the result of (13) is quantized to \pm 1 to obtain an estimation of $s_{k}[2 i]$, similarly, $s_{k}[2 i+1]$ also can be estimated by the same procedure.

\section{Modulation Multiplexing DSTC with Selection Relay (S-DSTC-MM)}

In the cooperative diversity system, the relaying protocol is a crucial component to achieve diversity. In [2], Selection Relay (SR) was proposed in which not 
all relay mobiles are allowed to transmit the relay signals. The permission to transmit the relay signals or not depends on the amplitudes of the fading gains between the user and its partner. If these values fall below a certain threshold, the relay user is not allowed to transmit the relay signal. In this paper, we also adopt SR as a relaying protocol, but the permission criteria is changed into the signal to noise ratio (SNR) at the output of the receiver. For easy description, we rewrite the received signal vector (9) based on several switching parameters $\left(\rho_{1}, \rho_{2}, \theta_{1}, \theta_{2}\right)$ as follows:

$$
\begin{aligned}
\hat{\mathbf{r}}[i+1]= & \left(\begin{array}{c}
\hat{r}_{B}[2 i+2] \\
\hat{r}_{B}[2 i+3]
\end{array}\right)=\left(\left|h_{1 B}[i+1] \rho_{2} h_{2}[i]\right|^{2} a_{1}+\left|h_{2 B}[i+1] \rho_{1} h_{1}[i]\right|^{2} a_{2}\right) \\
& \cdot\left(\begin{array}{c}
\theta_{1} s_{1}[2 i]+\theta_{2} j s_{2}[2 i+1] \\
\left(\theta_{1} s_{1}[2 i+1]+\theta_{2} j s_{2}[2 i]\right)^{*}
\end{array}\right)+\mathbf{H}^{H} \cdot \text { Noise }
\end{aligned}
$$

where

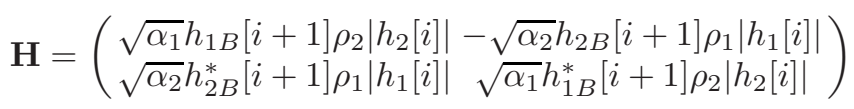

Based on these parameters, there exist three cases:

1. only the first user transmit the relay information: in this case, the first user acts as a relay and only transmits the relay information, and switching parameters are set as $\rho_{1}\left|h_{1}[i]\right|=1, \rho_{2}=1, \theta_{1}=0$ and $\theta_{2}=1$. Hence the SNR at the output of the receiver for the second user is shown as follows:

$$
S N R_{1}=\frac{a_{1}\left|h_{1 B}[i+1] h_{2}[i]\right|^{2}+a_{2}\left|h_{2 B}[i+1]\right|^{2}}{\left(a_{1}\left|h_{1 B}[i+1]\right|^{2}+1\right) \cdot N}
$$

2. only the second user transmits relay information: in this case, the roles of the first user and second user is switched. Parameters are given as $\rho_{1}=$ $1, \rho_{2}\left|h_{2}[i]\right|=1, \theta_{1}=1$ and $\theta_{2}=0$ and the SNR for the first user is

$$
S N R_{2}=\frac{a_{2}\left|h_{2 B}[i+1] h_{1}[i]\right|^{2}+a_{1}\left|h_{1 B}[i+1]\right|^{2}}{\left(a_{2}\left|h_{2 B}[i+1]\right|^{2}+1\right) \cdot N}
$$

3. both users transmit relay information: both users act as relays and parameters are set as $\rho_{1}=1, \rho_{2}=1, \theta_{1}=1$ and $\theta_{2}=1$. The SNR for the $i$ th user is shown as follows:

$$
S N R_{3}=\frac{a_{u}\left|h_{u B}[i+1] h_{v}[i]\right|^{2}+a_{v}\left|h_{v B}[i+1] h_{u}[i]\right|^{2}}{\left(a_{u}\left|h_{u B}[i+1]\right|^{2}+a_{v}\left|h_{v B}[i+1]\right|^{2}+1\right) \cdot N}
$$

where $u, v \in\{1,2\}, u \neq v$.

The SR is applied by comparing these three SNRs at the base station. When $S N R_{i}$ is the largest value among three SNRs, the corresponding case $i$ occurs. Hence the optimum SNR is $S N R_{\text {opt }}=\max \left\{S N R_{i}\right\}, i \in 1,2,3$. 


\section{Results and Discussion}

We assume users experience symmetric inter-user channel. Performance of the proposed DSTC-MM system, the amplify-and-forward system (AAF) [3] and the conventional DSTC system with TDMA [6] are evaluated.

\subsection{DSTC-MM Without SR}

In Fig 3, the DSTC-MM system achieves a performance gain over the AAF system 4 in the medium range of EbNo when the SNR of inter-user channel defined as isnr is larger than 10dB. The DSTC-MM achieves the almost same diversity gain as that of the DSTC system. However, it loses about 3-dB performance gain compared with the conventional DSTC at BER of 10-3 whether isnr is $20 \mathrm{~dB}$ or $30 \mathrm{~dB}$. Because the proposed scheme use the relay information and the user's information to construct a 2MQAM signal but the conventional DSTC applies MPAM modulation.

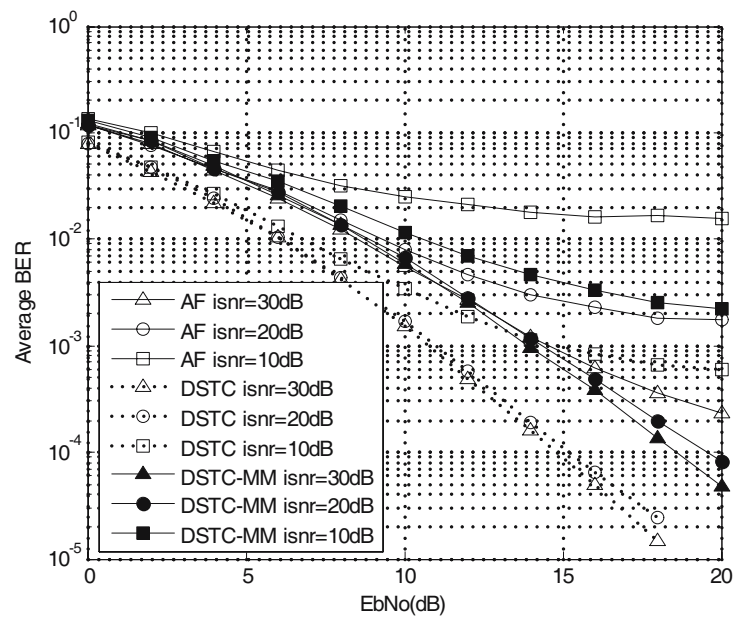

Fig. 3. BER comparison among the DSTC, the AAF and the DSTC-MM systems

\subsection{DSTC-MM with SR (S-DSTC-MM)}

It is important to establish how the selection relay affects the DSTC-MM system. And the performance of the S-DSTC-MM system is shown in Fig 4 . All BER curves of the S-DSTC-MM system have the same slope as that of the DSTC-MM system, which means that the S-DSTC-MM system achieves the same spatial diversity as that of the DSTC-MM system. Moreover, the S-DSTC-MM system achieves 3dB coding gain compared to the DSTC-MM system at the BER of $10^{-3}$. 


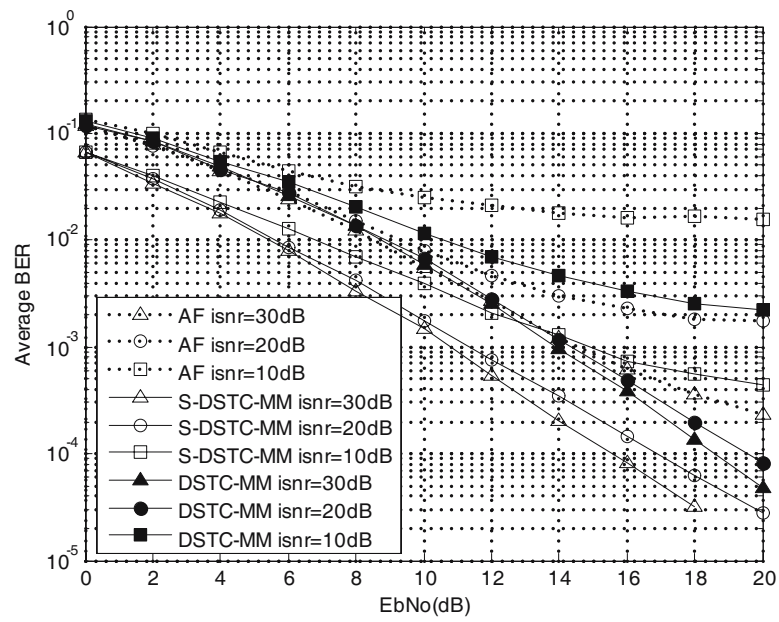

Fig. 4. BER comparison among the S-DSTC-MM, the AAF and the DSTC-MM systems

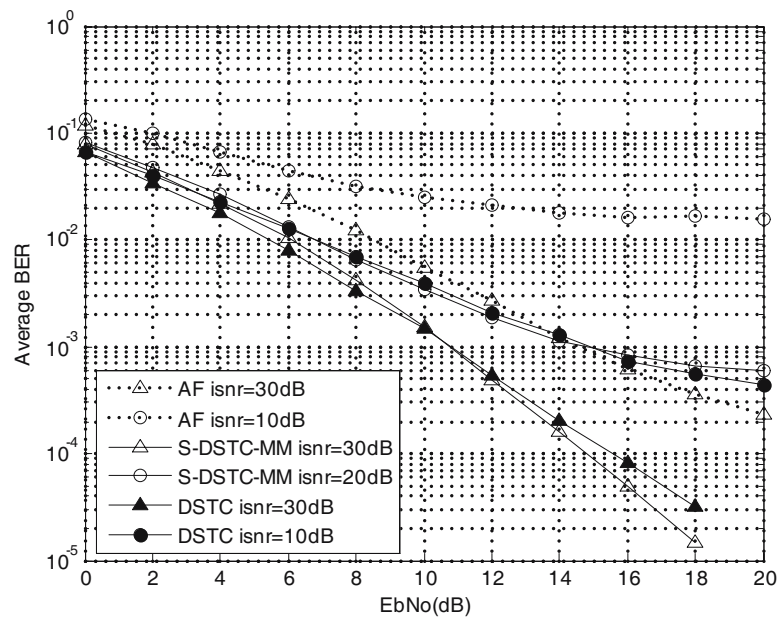

Fig. 5. BER comparison among the DSTC, the AAF and the S-DSTC-MM systems

Performances of the S-DSTC-MM and the conventional DSTC systems are evaluated in Fig.5. When isnr $=10 \mathrm{~dB}$, performances of the S-DSTC-MM system is as good as that of the DSTC system. When isnr $=30 d B$, the S-DSTCMM system outperforms the conventional DSTC system at medium range of high EbNo. Furthermore, The S-DSTC-MM system achieves much better performance gain than the AAF system when the inter-user channel is bad such as $i s n r=10 \mathrm{~dB}$. 


\section{Conclusion}

In this paper, we propose a modulation multiplexing distributed space-time coding for two-user cooperative diversity system in wireless network. In the system, the information of the first user lies in $\mathbf{I}$ axis, while the information of the second user lies in $\mathbf{Q}$ axis, which ensures both users can share time and frequency resource during the cooperation frame and increases the bandwidth efficiency. We also adopt the selection relay protocol for the proposed system to improve the coding gain. Based on simulation results, we conclude that the proposed DSTC-MM system significantly outperforms the AAF cooperative system when inter-user channel SNR is high. Furthermore, the scheme achieves the same diversity as that of the DSTC cooperative system in [4. However, around 3-dB coding gain is lost. By applying selection relaying, the S-DTSC-MM system outperforms the DSTC system at medium range of high EbNo.

Acknowledgments. This research was supported by the Brain Korea 21 project and also supported by the Institute Information Technology Assessment (IITA) of the Ministry of Information and Communication of Korea Government.

\section{References}

1. Sendonaris, A., Erkop, E., Aazhang, B.: User cooperation diveristy -Part I: system description. IEEE Trans. Commun. 51, 1927-1938 (2003)

2. Laneman, J.N., Tse, D.N.C., Wornell, G.W.: Cooperative diversity in wireless networks: efficience protocols and outage behavior. IEEE Trans. Inf. Theory 50(12), 3062-3080 (2004)

3. Laneman, J.N., Wornell, G.W.: Energy-efficient antenna sharing and relaying for wireless networks. Proc. of wireless communication and networking conference 1, 7-12 (2000)

4. Laneman, J.N., Wornell, G.W.: Distributed space-time coded protocols for exploiting cooperative diversity in wireless networks. In: Proc. of Clobal Telecom. conference, vol. 1, pp. 77-81 (2002)

5. Anghel, P.A., Leus, G., Kaveh, M.: Distributed space-time coding in cooperative networks. In: Proc. of Intenational Conference on speech and signal processing (April 2003)

6. Laneman, J.N., Wornell, G.W.: Distributed space-time coded protocols for exploiting cooperative diversity in wireless networks. IEEE Trans. Information Theory 49, 2415-2425 (2003)

7. Chang, Y., Hua, Y.: Diversity analysis of orthogonal space-time modulation for distributed wireless relays. In: Proc. of International conference on Acoustics, Speech and Signal Proc., May 2004, vol. 4, pp. 561-564 (2004) 